\title{
Assessment of drought tolerance in various cotton genotypes under simulated osmotic settings
}

\author{
Muhammad Riaz Gondal1*, Muhammad Yasir Saleem², Sultan Ahmad Rizvi1, Aaqib Riaz ${ }^{3}$, Waqas Naseem1, \\ Ghulam Muhammad1, Sikandar Hayat4, Mazher Iqbal ${ }^{5}$ \\ ${ }^{1}$ Soil and Water Conservation Research Institute, Chakwal, Pakistan \\ ${ }^{2}$ Fauji Fresh and Freeze, FFC Limited, Sahiwal, Pakistan \\ ${ }^{3}$ Hussain Park, Military Farm Road, Sargodha, Pakistan \\ ${ }^{4}$ Fodder Research Institute, Sargodha, Pakistan \\ ${ }^{5}$ Barani Agricultural Research Institute, Chakwal, Pakistan
}

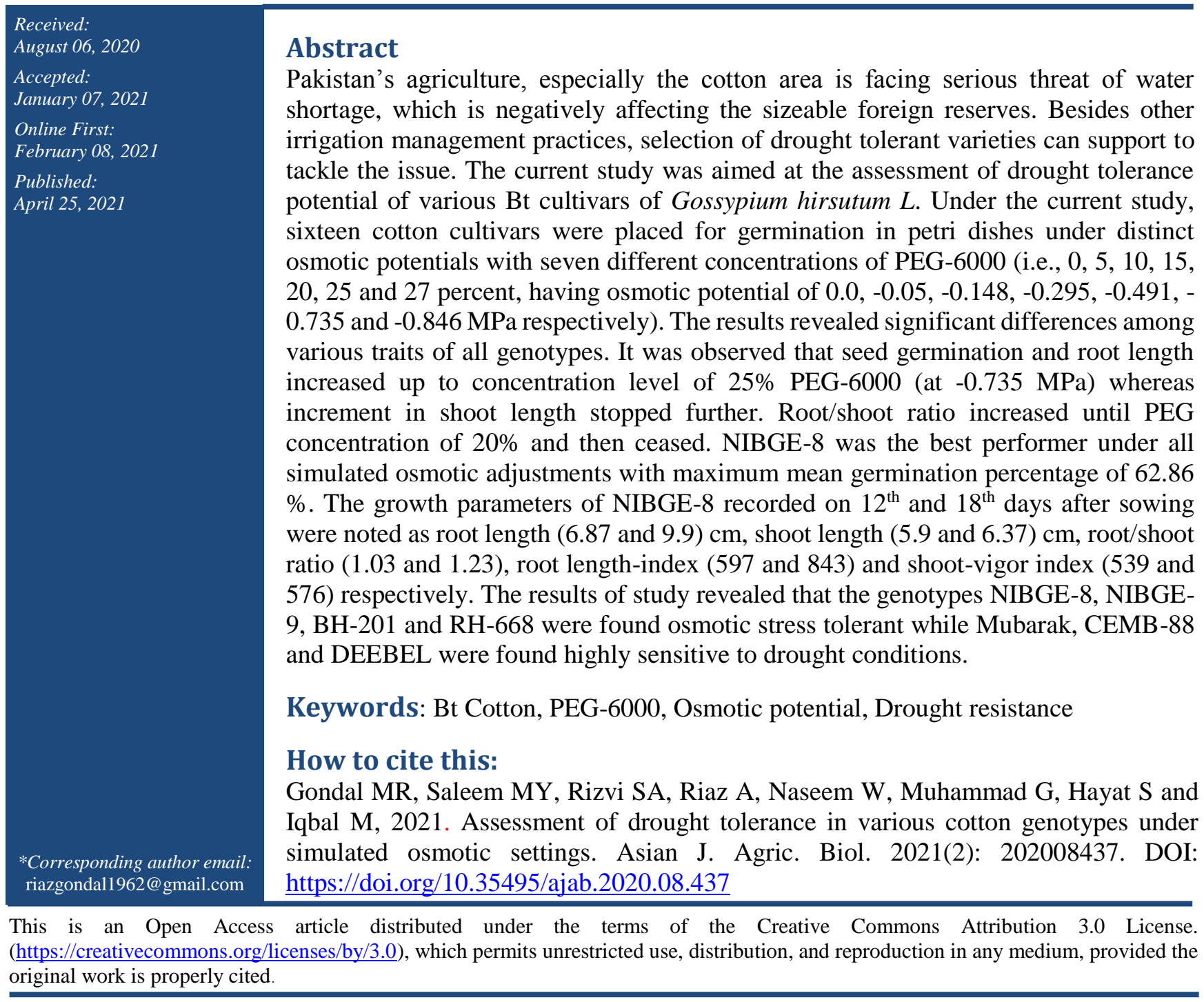




\section{Introduction}

Cotton (Gossypium hirsutum L.) is the essential fiber crop of the world, highly demanded all over the world. Nearly 70 nations of the world grow cotton for their domestic use and/or for export purposes. It is grown mostly in warm as well as mild zones of the world. During 2019-20, cotton was cultivated on about 2.5 million hectares, having annual production of 9.86 million bales (480 lb, bale) with average yield of 618 $\mathrm{kg} \mathrm{ha}^{-1}$ (Economic Survey of Pakistan, 2020). Its contribution in GDP is $0.8 \%$ while its share in agriculture value addition is $4.1 \%$. Harvesting of cotton is done by picking the cotton bolls from which seed is separated after ginning. Seed is utilized for extraction of oil and pressed seed is used for feeding livestock. Remaining fiber known as 'lint' is the entity of great importance which is further processed for the production of thread and ultimately for outfits production.

Large yield gaps exist between production and yield potentials. These yield gaps are normally due to poor cultural practices, shortage of quality seed and lack of inputs (fertilizer, insecticide and irrigation water) as well as biotic and abiotic stress especially the drastic environmental influences such as extreme drought and high temperature regimes. It is said that in future cotton will have to be grown under extreme water shortage and high temperatures in relation with other abiotic stresses (Dabbert and Gore, 2014). Cotton growing areas in Pakistan are mostly warm and arid regions having little rainfall in addition to high temperature during season of cotton (Riaz et al., 2013). Cotton yield depends upon various factors such as potential of cultivar, environmental fluctuations and overall cultural practices. These factors influence the crop individually as well as in combination, which ultimately decreases yield significantly (Romagosa and Fox, 1993).

Abiotic stresses especially drought and heat mutually creates adverse survival circumstances in the life cycle of cotton crop plant. Cotton crop has the ability to withstand under drought conditions but for higher yields it need adequate water supply of about 2,158 to $3,906 \mathrm{~m}^{3}$ every growing season (McWilliams, 2003). Thus, yield and production of cotton increases with intense rainfall pattern. Few developmental stages such as flowering initiation stage and boll developing phase require sufficient water supply (McWilliams, 2003). Plant needs water at different developmental stages during its life cycle, which critically depends on plant water losses via transpiration and moisture quantity present in soil (Allen et al., 1998). Similarly plant developmental stage and time of irrigation as well as drought situation during cotton growing period, states the up and downs in the yield (Boman and Lemon, 2006). If drought stress remains for longer periods it effects plant height due to which short statured plant could be seen in field as compared to plant having sufficient water supply (Pace et al., 1999). Cotton boll weight, formation of seed inside the boll, seed and lint index as well as staple size, consistency, fiber development and its longevity are highly influenced under water stress circumstances (Wen et al., 2013).

Though cotton genotypes are significantly adjusted in particular environment and their selection is done after observing maximum potential in accordance to each specific desirable trait but, when it comes to shortage of water, these overwhelming challenges become hurdles in achieving maximum lint and seed yield which ultimately decreases the overall production. Survival of crop plant and increment in yield under such circumstances can be achieved by cultural and management practices such as by raising cover crops, sowing on beds, adopting zero tillage and using high efficiency irrigation practices to maintain soil moisture and tackle the water shortage at crucial stages. Besides these practices, selection of drought tolerant variety is vital to cover the gap of production. Keeping in view, these obstacles in cotton yield, the current study was planned to assess the drought tolerance potential of various Bt cotton cultivars using osmotic concentration technique.

\section{Material and Methods}

\section{Experimental site and design}

The experimental study was conducted to determine the effect of drought stress on yield and yield attributes of various cotton cultivars in the Laboratory located at the Department of Plant Breeding and Genetics, Faculty of Agriculture, Gomal University D.I Khan during the year 2017-18. The experiment was laid out under completely randomized design (CRD) having factorial arrangement with three replications.

\section{Treatments and data recording}

Sixteen cotton cultivars viz., BH-201, CEMB-55, CEMB-88, CIM-602, CIM-625, CIM-632, CYTO179, CYTO-313, DEEBEL, FH-142, FH-152, FH- 
326, Mubarak, NIBGE-8, NIBGE-9 and RH-668 were selected for assessment of their tolerance against drought.

Ten healthy, identical and lint free seeds of each cultivar were chosen. These seeds were placed for germination, one at bottom and other on top of filter papers in sterilized petri dishes to maintain concentrations at $27^{\circ} \mathrm{C}$. The seeds were placed apart in each petri dish under seven different concentrations levels of PEG-6000 i.e., 0, 5, 10, 15, 20, 25 and 27\% that generated osmotic potential of $0.0,-0.05,-0.148$, $-0.295,-0.491,-0.735$ and $-0.846 \mathrm{MPa}$ respectively. The concentrations were applied in such a way that 2 $\mathrm{mL}$ of each concentration at bottom and $1 \mathrm{~mL}$ on upper side of filter paper were applied. Then the petri dishes were transferred to incubator having temperature set at $27^{\circ} \mathrm{C}$. In order to maintain the levels of osmotic potential each PEG-6000 concentration was applied to petri dishes after an interval of 48-72 hours. The PEG6000 concentration required to achieve a specific osmotic potential was computed by the formula given by Michel and Kaufmann (1973) expressed as:

$$
\begin{gathered}
\Psi \mathrm{s}=-\left(1.18 \times 10^{-2}\right) \times C-\left(1.18 \times 10^{-4}\right) \times C_{2}+\left(2.67 \times 10^{-4}\right) \times C \\
\times T+\left(8.39 \times 10^{-7}\right) \times C_{2} \times T
\end{gathered}
$$

Where:

$\Psi_{\mathrm{s}}=$ osmotic potential (bar);

$\mathrm{C}=$ concentration $\left(\mathrm{g} \mathrm{L}^{-1}\right.$ PEG-6000 in water $)$;

$\mathrm{T}=$ temperature $\left({ }^{\circ} \mathrm{C}\right)$.

For control, a solution with osmotic potential $\Psi \mathrm{s}=0.0$ MPa was used.

All the treatments were observed for germination (\%), shoot length $(\mathrm{cm})$ and root length $(\mathrm{cm})$ recorded at $12^{\text {th }}$ and $18^{\text {th }}$ day after sowing (DAS) and implementation of PEG-6000 concentrations. Mean values of all three replications for each of the parameters were calculated and presented in data tables. Various plants growth parameters were investigated as described below:

Germination ( $\%$ age): Sprouted seed in each petri dish were considered as germinated seed and counted on $12^{\text {th }}$ and $18^{\text {th }}$ day after sowing. The germination percentage was computed as follows:

Germination $(\%)=\left(\frac{\text { Number of germinated seed }}{\text { Number of seed sown }}\right) \times 100$

Root and shoot length (cm): Five seedlings were taken out at random from each petri-dish without disturbing the root of seedling on $12^{\text {th }}$ and $18^{\text {th }}$ day after sowing and the longest root was measured in centimeters from the collar to the tip and recorded. Similarly, the shoot lengths were also measured for each treatment and recorded accordingly.

Root to shoot ratio: Root lengths and shoot lengths recorded on $12^{\text {th }}$ and $18^{\text {th }}$ day after sowing were converted into root-shoot ratio as follows:

Root-shoot Ratio $=\frac{\text { Root length }}{\text { Shoot length }}$

Shoot and root vigor index: Shoot and root vigor indices were also computed at $12^{\text {th }}$ and $18^{\text {th }}$ day after sowing using the expression proposed by Abdul- Baki and Anderson, (1973).

Root length index $(\mathrm{RLI})=$ Root length $\times$ Germination $\%$

Shoot length index (SLI) $=$ Shoot length $\times$ Germination \%

\section{Statistical analysis}

All the data gathered were subjected to statistical analysis by following Fisher's analysis of variance method (Steel, 1997). Means were compared with LSD test at $5 \%$ probability level as described by Gomez and Gomez (1984).

\section{Results}

All the data collected under the study were analyzed statistically. The calculated variances showed highly significant differences among the genotypes as well as the interactive effects among all the parameters investigated. These results are presented in following tables (Table-1 \& Table-2).

Table-1. Analysis of variance for germination percentage

\begin{tabular}{l|c|c|c|c|c|}
\hline \multicolumn{1}{|c|}{ Source } & DF & SS & MS & F \\
\hline Genotype & 15 & 8871.43 & 591.429 & $6.4 \mathrm{E}+30 * *$ \\
\hline PEG & 6 & 473239 & 78873.2 & $8.5 \mathrm{E}+32 * *$ \\
\hline Genotype*PEG & 90 & 6503.57 & 72.2619 & $7.8 \mathrm{E}+29 * *$ \\
\hline Error & 224 & $2.09 \mathrm{E}-26$ & $9.31 \mathrm{E}-29$ & \\
\hline Total & 335 & 488614 & & \\
** means highly significant
\end{tabular}


Muhammad Riaz Gondal et al.

Table-2. Mean squares for various seedling related traits

\begin{tabular}{|l|c|c|c|c|c|c|}
\hline \multicolumn{1}{|c|}{ Source } & DF & RL & SL & RSR & RLI & SVI \\
\hline Genotype & 15 & $40.5528^{* *}$ & $15.4278^{* *}$ & $0.12113^{* *}$ & $381373^{* *}$ & $162312^{* *}$ \\
\hline PEG & 6 & $2622.59^{* *}$ & $1942.94^{* *}$ & $78.9533^{* *}$ & $21190000^{* *}$ & $18510000^{* *}$ \\
\hline Growth stage & 1 & $1173.43^{* *}$ & $161.171^{* *}$ & $0.08371^{* *}$ & $6717600^{* *}$ & $848806^{* *}$ \\
\hline Genotype*PEG & 90 & $2.43476^{* *}$ & $1.35541^{* *}$ & $0.09697^{* *}$ & $36570.8^{* *}$ & $18408.1^{* *}$ \\
\hline Genotype*Growth stage & 15 & $0.28686^{* *}$ & $0.82599^{* *}$ & $0.07628^{* *}$ & $5162.84^{* *}$ & $4589.14^{* *}$ \\
\hline PEG* Growth stage & 6 & $82.382^{* *}$ & $24.533^{* *}$ & $7.28871^{* *}$ & $735968^{* *}$ & $175237^{* *}$ \\
\hline Genotype*Growth- stage*PEG & 90 & $0.18196^{* *}$ & $0.32518^{* *}$ & $0.06594^{* *}$ & $1823.59^{* *}$ & $1867.36^{* *}$ \\
\hline Error & 448 & $2.26 \mathrm{E}-30$ & $9.95 \mathrm{E}-31$ & $5.41 \mathrm{E}-32$ & $1.27 \mathrm{E}-26$ & $1.25 \mathrm{E}-26$ \\
\hline Total & 671 & & & & & \\
\hline
\end{tabular}

** means highly significant

\section{Germination percentage}

It was observed that the germination percentage reduces as the PEG-6000 concentration increases and became zero at concentration level of $27 \%$. Mean germination values at PEG-6000 concentrations levels of $0 \%, 5 \%, 10 \%, 15 \%, 20 \%, 25 \%$ and $27 \%$ which generated osmotic potentials of $0.0 \mathrm{MPa},-0.05 \mathrm{MPa}$, $0.148 \mathrm{MP},-0.295 \mathrm{MPa}, 0.491 \mathrm{MPa},-0.735 \mathrm{MPa}$ and $0.846 \mathrm{MPa}$ respectively were observed as $100 \%$, $89.375 \%, 80.625 \%, 66.875 \%, 35 \%, 5.625 \%$ and $0 \%$ respectively (Table-3).

Table-3. Effect of different concentrations of PEG6000 on germination \%age of various cultivars

\begin{tabular}{|l|c|c|c|c|c|c|c|c|}
\hline \multirow{2}{*}{ Genotype } & \multicolumn{9}{|c|}{ Seed germination (\%) at various PEG Concentrations } \\
\cline { 2 - 10 } & $\mathbf{0 \%}$ & $\mathbf{5 \%}$ & $\mathbf{1 0 \%}$ & $\mathbf{1 5 \%}$ & $\mathbf{2 0 \%}$ & $\mathbf{2 5 \%}$ & $\mathbf{2 7 \%}$ & Mean \\
\hline BH-201 & 100 & 90 & 80 & 70 & 40 & 10 & 0 & 55.71 \\
\hline CEMB-55 & 100 & 80 & 80 & 70 & 40 & 0 & 0 & 52.86 \\
\hline CEMB-88 & 100 & 90 & 80 & 70 & 40 & 10 & 0 & 55.71 \\
\hline CIM-602 & 100 & 80 & 70 & 60 & 30 & 0 & 0 & 48.57 \\
\hline CIM-625 & 100 & 80 & 80 & 60 & 20 & 0 & 0 & 48.57 \\
\hline CIM-632 & 100 & 90 & 80 & 70 & 40 & 10 & 0 & 55.71 \\
\hline CYTO-179 & 100 & 90 & 80 & 60 & 20 & 0 & 0 & 50.00 \\
\hline CYTO-313 & 100 & 80 & 70 & 50 & 20 & 0 & 0 & 45.71 \\
\hline DEEBEL & 100 & 100 & 90 & 80 & 50 & 20 & 0 & 62.86 \\
\hline FH-142 & 100 & 90 & 80 & 70 & 30 & 0 & 0 & 52.86 \\
\hline FH-152 & 100 & 90 & 80 & 70 & 40 & 0 & 0 & 54.29 \\
\hline FH-326 & 100 & 90 & 80 & 70 & 40 & 0 & 0 & 54.29 \\
\hline Mubarak & 100 & 80 & 70 & 50 & 20 & 0 & 0 & 45.71 \\
\hline NIBGE-8 & 100 & 100 & 90 & 80 & 50 & 20 & 0 & 62.86 \\
\hline NIBGE-9 & 100 & 100 & 90 & 70 & 40 & 10 & 0 & 58.57 \\
\hline RH-668 & 100 & 100 & 90 & 70 & 40 & 10 & 0 & 58.57 \\
\hline Mean & 100 & 89.4 & 80.6 & 66.9 & 35 & 5.63 & 0 & 53.93 \\
\hline Alpha 0.05 & \multicolumn{9}{|c|}{ Standard Error for Comparison =1.4045 } \\
\hline
\end{tabular}

The results showed that the highest mean germination percentage under all PEG-6000 concentration was observed in the genotypes NIBGE-8 and DEEBEL having at par value of $62.86 \%$, followed by NIBGE-9 and RH-668 with germination percentage of (58.57\%), while the lowest germination percentage was shown by CYTO-313 and Mubarak with statistically at par value of $45.71 \%$. From these results, it is concluded that all cotton genotypes can germinate up to $20 \%$ (0.491 MPa) concentration of PEG-6000.

\section{Root length}

The results showed that increment in root length was observed until PEG-6000 concentration of $10 \%$, beyond that level root length was reduced leading to complete cessation at concentration level of $27 \%$. Maximum root length $(12.1 \mathrm{~cm} \& 14.8 \mathrm{~cm})$ was observed at $-0.148 \mathrm{MPa}$ under both growth stages (after $12^{\text {th }} \& 18^{\text {th }}$ DAS) (Table-4). Under entire concentrations of PEG-6000, NIBGE-8 was the best performer with maximum root length $(9.9 \mathrm{~cm})$ succeeded by NIBGE-9 $(9.19 \mathrm{~cm})$ while Mubarak was the least performer with minimum root length $(6.26$ $\mathrm{cm})$.

\section{Shoot length}

Analysis of shoot length data showed inverse relationship among PEG-6000 concentrations and the shoot length in all genotypes i.e., the shoot length decreased with rise of PEG-6000 concentration. On $18^{\text {th }}$ day after sowing the mean shoot lengths under all concentrations of PEG-6000 (i.e. 0, 5, 10, 15, 20, 25 and $27 \%$ ) were observed as $11.98,10.32,8.38,5.64$, $3.28,0$ and $0 \mathrm{~cm}$ respectively (Table-5). Maximum mean shoot length was observed in genotype NIBGE$8(6.37 \mathrm{~cm})$ followed by NIBGE-9 $(6.24 \mathrm{~cm})$ and lowest shoot length was recorded in genotype Mubarak $(4.67 \mathrm{~cm})$. The shoot length was completely ceased at $25 \%$ concentration having osmotic potential of $-0.735 \mathrm{MPa}$. 
Muhammad Riaz Gondal et al.

Table-4. Effect of different concentrations of PEG-6000 on root length $(\mathrm{cm})$ at two growth stages

\begin{tabular}{|c|c|c|c|c|c|c|c|c|c|c|c|c|c|c|c|c|}
\hline \multirow{2}{*}{ Genotype } & \multicolumn{8}{|c|}{ PEG-6000 concentration on $12^{\text {th }}$ DAS } & \multicolumn{8}{|c|}{ PEG-6000 concentration on $18^{\text {th }}$ DAS } \\
\hline & $0 \%$ & $5 \%$ & $10 \%$ & $15 \%$ & $20 \%$ & $25 \%$ & $27 \%$ & Mean & $0 \%$ & $5 \%$ & $10 \%$ & $15 \%$ & $20 \%$ & $25 \%$ & $27 \%$ & Mean \\
\hline BH-201 & 8.4 & 9.3 & 13.7 & 9 & 5 & 0.3 & 0 & 6.53 & 12.2 & 13.6 & 15.7 & 13 & 5.7 & 0.6 & 0 & 8.69 \\
\hline CEMB-55 & 6.3 & 7.2 & 11.1 & 6.9 & 2.5 & 0 & 0 & 4.86 & 10.8 & 12.1 & 14.3 & 10.6 & 4 & 0 & 0 & 7.4 \\
\hline CEMB-88 & 5.3 & 6.5 & 10.1 & 6.1 & 1.9 & 0.1 & 0 & 4.29 & 9.4 & 10.9 & 13 & 9.1 & 4.5 & 0.2 & 0 & 6.73 \\
\hline CIM-602 & 8.2 & 9.1 & 13 & 8.6 & 4.5 & 0.2 & 0 & 6.23 & 12.1 & 13.5 & 15.5 & 13.1 & 6.7 & 0.5 & 0 & 8.77 \\
\hline CIM-625 & 8 & 8.9 & 12.9 & 8.3 & 4.4 & 0.1 & 0 & 6.09 & 12 & 13.3 & 15.5 & 12.9 & 7.1 & 0.2 & 0 & 8.71 \\
\hline CIM-632 & 7.5 & 8.2 & 12.2 & 8.1 & 4 & 0.1 & 0 & 5.73 & 11.4 & 12.8 & 15 & 12.6 & 6.3 & 0.2 & 0 & 8.33 \\
\hline CYTO-179 & 5.7 & 6.9 & 10.8 & 6.6 & 2.2 & 0 & 0 & 4.6 & 10.2 & 11.7 & 13.5 & 9.8 & 4.7 & 0 & 0 & 7.13 \\
\hline CYTO-313 & 5.9 & 7 & 11.1 & 6.7 & 2.4 & 0 & 0 & 4.73 & 10.3 & 11.7 & 13.8 & 10.1 & 5.4 & 0 & 0 & 7.33 \\
\hline DEEBEL & 5.5 & 6.8 & 10.5 & 6.3 & 2 & 0.1 & 0 & 4.46 & 9.9 & 11.4 & 13.4 & 9.4 & 4.5 & 0.3 & 0 & 6.99 \\
\hline FH-142 & 7.7 & 8.4 & 12.3 & 8.2 & 4.1 & 0 & 0 & 5.81 & 11.4 & 12.9 & 15.1 & 12.4 & 6.2 & 0 & 0 & 8.29 \\
\hline FH-152 & 7.5 & 8.1 & 12 & 8.1 & 3.9 & 0 & 0 & 5.66 & 11.3 & 12.4 & 14.7 & 12 & 5.9 & 0 & 0 & 8.04 \\
\hline FH-326 & 7.9 & 8.7 & 12.7 & 8.3 & 4.2 & 0 & 0 & 5.97 & 11.7 & 13.1 & 15.3 & 12.8 & 6.1 & 0 & 0 & 8.43 \\
\hline Mubarak & 4.7 & 5.8 & 9.9 & 5.4 & 1.2 & 0 & 0 & 3.86 & 9.3 & 10.5 & 12.7 & 8.7 & 2.6 & 0 & 0 & 6.26 \\
\hline NIBGE-8 & 8.7 & 9.9 & 14.3 & 9.4 & 5.4 & 0.4 & 0 & 6.87 & 12.9 & 14.4 & 17.1 & 14.1 & 9.5 & 1.3 & 0 & 9.9 \\
\hline NIBGE-9 & 8.5 & 9.5 & 13.9 & 9.1 & 5 & 0.2 & 0 & 6.6 & 12.5 & 14 & 16.5 & 13.6 & 8.6 & 0.9 & 0 & 9.44 \\
\hline RH-668 & 8.2 & 9.2 & 13.4 & 8.7 & 4.9 & 0.2 & 0 & 6.37 & 12.3 & 13.8 & 15.8 & 13.3 & 8.4 & 0.7 & 0 & 9.19 \\
\hline Mean & 7.13 & 8.09 & 12.1 & 7.74 & 3.6 & 0.11 & 0 & 5.54 & 11.2 & 12.6 & 14.8 & 11.7 & 6.01 & 0.31 & 0 & 8.1 \\
\hline Alpha 0.05 & \multicolumn{8}{|c|}{ Standard Error for Comparison $=0.0381$} & \multicolumn{8}{|c|}{ LSD $=0.1304$} \\
\hline
\end{tabular}

Table-5. Effect of different concentrations of PEG-6000 on cotton shoot length $(\mathrm{cm})$ at different growth stages

\begin{tabular}{|c|c|c|c|c|c|c|c|c|c|c|c|c|c|c|c|c|}
\hline \multirow{2}{*}{ Genotype } & \multicolumn{8}{|c|}{ PEG-6000 concentration on $12^{\text {th }}$ day after sowing } & \multicolumn{8}{|c|}{ PEG-6000 concentration on $18^{\text {th }}$ day after sowing } \\
\hline & 0\% & $5 \%$ & $10 \%$ & $15 \%$ & $20 \%$ & $25 \%$ & $27 \%$ & Mean & $\mathbf{0 \%}$ & $5 \%$ & $10 \%$ & $15 \%$ & $20 \%$ & $25 \%$ & $27 \%$ & Mean \\
\hline BH-201 & 10.9 & 9.9 & 8 & 7.2 & 1.6 & 0 & 0 & 5.37 & 12.7 & 10.9 & 9 & 6.5 & 3.9 & 0 & 0 & 6.14 \\
\hline CEMB-55 & 9 & 8.7 & 6.9 & 4.5 & 0.9 & 0 & 0 & 4.29 & 11.6 & 10 & 8.1 & 5.2 & 3.1 & 0 & 0 & 5.43 \\
\hline CEMB-88 & 8.3 & 6.9 & 5.1 & 3.9 & 0.7 & 0 & 0 & 3.56 & 10.9 & 9.5 & 7.4 & 4.5 & 2.9 & 0 & 0 & 5.03 \\
\hline CIM-602 & 10.5 & 9.9 & 8.2 & 7.7 & 1.8 & 0 & 0 & 5.44 & 12.5 & 10.9 & 8.9 & 6.2 & 4.2 & 0 & 0 & 6.1 \\
\hline CIM-625 & 10 & 9.4 & 7.9 & 6.6 & 1.5 & 0 & 0 & 5.06 & 12.3 & 10.7 & 8.9 & 6.1 & 4.1 & 0 & 0 & 6.01 \\
\hline CIM-632 & 9.6 & 9.1 & 7.5 & 6.1 & 1.3 & 0 & 0 & 4.8 & 12 & 10.5 & 8.6 & 5.7 & 3.2 & 0 & 0 & 5.71 \\
\hline CYTO-179 & 8.7 & 8.2 & 6.4 & 4.1 & 0.9 & 0 & 0 & 4.04 & 11.4 & 9.8 & 7.6 & 4.8 & 2.6 & 0 & 0 & 5.17 \\
\hline CYTO-313 & 8.9 & 8.4 & 6.6 & 4.2 & 1 & 0 & 0 & 4.16 & 11.6 & 10.1 & 8.4 & 5.5 & 2.7 & 0 & 0 & 5.47 \\
\hline DEEBEL & 8.5 & 8.1 & 6.4 & 4.1 & 0.8 & 0 & 0 & 3.99 & 11.2 & 9.7 & 7.4 & 4.7 & 3 & 0 & 0 & 5.14 \\
\hline FH-142 & 9.8 & 9.8 & 7.2 & 5.9 & 1.1 & 0 & 0 & 4.83 & 12.2 & 10.5 & 8.8 & 6 & 3.4 & 0 & 0 & 5.84 \\
\hline FH-152 & 9.7 & 9.7 & 7.1 & 5.7 & 1 & 0 & 0 & 4.74 & 12 & 10.2 & 8.5 & 5.7 & 3.1 & 0 & 0 & 5.64 \\
\hline FH-326 & 9.9 & 9.8 & 7.4 & 6.1 & 1.4 & 0 & 0 & 4.94 & 12.1 & 10.2 & 8.3 & 5.4 & 3.1 & 0 & 0 & 5.59 \\
\hline Mubarak & 7.9 & 6.7 & 4.4 & 3.5 & 0.5 & 0 & 0 & 3.29 & 10.5 & 9.2 & 7.1 & 4.4 & 1.5 & 0 & 0 & 4.67 \\
\hline NIBGE-8 & 11.9 & 10.4 & 8.3 & 8.7 & 2 & 0 & 0 & 5.9 & 13.3 & 11.3 & 9.2 & 6.7 & 4.1 & 0 & 0 & 6.37 \\
\hline NIBGE-9 & 11.5 & 10.1 & 8 & 7.3 & 1.5 & 0 & 0 & 5.49 & 13 & 11.1 & 9.1 & 6.5 & 4 & 0 & 0 & 6.24 \\
\hline RH-668 & 10.4 & 9.8 & 7.3 & 6.2 & 1.4 & 0 & 0 & 5.01 & 12.4 & 10.6 & 8.9 & 6.4 & 3.7 & 0 & 0 & 6 \\
\hline Mean & 9.72 & 9.06 & 7.04 & 5.74 & 1.21 & 0 & 0 & 4.68 & 12 & 10.3 & 8.39 & 5.64 & 3.29 & 0 & 0 & 5.66 \\
\hline Alpha 0.05 & & Stan & dard E & ror fo & Comp & arison & $=0.050$ & & & & & $\mathrm{LSD}=$ & 0.174 & & & \\
\hline
\end{tabular}

\section{Root to shoot ratio}

The result from data analysis of root to shoot ratio revealed that ratio was increasing with increase of PEG6000 concentration. The maximum value of this ratio on $12^{\text {th }}$ DAS and $18^{\text {th }}$ DAS was observed at concentration levels of $20 \%$ and $15 \%$ respectively. There was a decreasing trend in root to shoot ratio on $18^{\text {th }}$ DAS at $20 \%$ concentration level while increasing trend at $15 \%$ concentration level. However, on $18^{\text {th }}$ DAS highest mean ratios were recorded for the genotypes NBGE-8 followed by RH-668 while minimum ratios were observed for cultivar BH-201. It is evident from results that maximum root to shoot ratio was recorded at 20\% PEG-6000 concentration on $12^{\text {th }}$ DAS followed by $10 \%$ concentration and minimum ratios were recorded at 25\%-27\% concentrations. Highest ratios on $18^{\text {th }}$ DAS were recorded at $15 \%$ concentration followed by $20 \%$ concentration while least ratios are recorded at 25-27\% concentration due to ceasing of root or shoot growth at these potentials (Table-6). 
Muhammad Riaz Gondal et al.

Table-6. Effect of different concentrations of PEG-6000 on cotton root to shoot ratio at different growth stages

\begin{tabular}{|c|c|c|c|c|c|c|c|c|c|c|c|c|c|c|c|c|}
\hline \multirow{2}{*}{ Genotype } & \multicolumn{8}{|c|}{ PEG-6000 concentration on $12^{\text {th }}$ day after sowing } & \multicolumn{8}{|c|}{ PEG-6000 concentration on $18^{\text {th }}$ day after sowing } \\
\hline & $0 \%$ & $5 \%$ & $10 \%$ & $15 \%$ & $20 \%$ & $25 \%$ & $27 \%$ & Mean & $0 \%$ & $5 \%$ & $10 \%$ & $15 \%$ & $20 \%$ & $25 \%$ & $27 \%$ & Mean \\
\hline BH-201 & 0.8 & 0.9 & 1.7 & 1.3 & 3.1 & 0 & 0 & 1.11 & 0.9 & 1.2 & 1.7 & 2 & 1.5 & 0 & 0 & 1.04 \\
\hline CEMB-55 & 0.7 & 0.8 & 1.6 & 1.5 & 2.8 & 0 & 0 & 1.06 & 0.9 & 1.2 & 1.8 & 2 & 1.5 & 0 & 0 & 1.06 \\
\hline CEMB-88 & 0.6 & 0.9 & 1.9 & 1.6 & 2.7 & 0 & 0 & 1.1 & 0.9 & 1.1 & 1.8 & 2 & 1.6 & 0 & 0 & 1.06 \\
\hline CIM-602 & 0.8 & 0.9 & 1.6 & 1.1 & 2.5 & 0 & 0 & 0.99 & 1 & 1.2 & 1.7 & 2.1 & 1.6 & 0 & 0 & 1.09 \\
\hline CIM-625 & 0.8 & 0.9 & 1.6 & 1.3 & 2.9 & 0 & 0 & 1.07 & 1 & 1.2 & 1.7 & 2.1 & 1.7 & 0 & 0 & 1.1 \\
\hline CIM-632 & 0.8 & 0.9 & 1.6 & 1.3 & 3.1 & 0 & 0 & 1.1 & 1 & 1.2 & 1.7 & 2.2 & 2 & 0 & 0 & 1.16 \\
\hline CYTO-179 & 0.7 & 0.8 & 1.7 & 1.6 & 2.4 & 0 & 0 & 1.03 & 0.9 & 1.2 & 1.8 & 2 & 1.8 & 0 & 0 & 1.1 \\
\hline CYTO-313 & 0.7 & 0.8 & 1.7 & 1.6 & 2.4 & 0 & 0 & 1.03 & 0.9 & 1.2 & 1.6 & 1.8 & 2 & 0 & 0 & 1.07 \\
\hline DEEBEL & 0.6 & 0.8 & 1.6 & 1.5 & 2.5 & 0 & 0 & 1 & 0.9 & 1.2 & 1.8 & 2 & 1.5 & 0 & 0 & 1.06 \\
\hline FH-142 & 0.8 & 0.9 & 1.7 & 1.4 & 3.7 & 0 & 0 & 1.21 & 0.9 & 1.2 & 1.7 & 2 & 1.8 & 0 & 0 & 1.09 \\
\hline FH-152 & 0.8 & 0.8 & 1.7 & 1.4 & 3.9 & 0 & 0 & 1.23 & 0.9 & 1.2 & 1.7 & 2.1 & 1.9 & 0 & 0 & 1.11 \\
\hline FH-326 & 0.8 & 0.9 & 1.7 & 1.4 & 3 & 0 & 0 & 1.11 & 1 & 1.3 & 1.8 & 2.4 & 2 & 0 & 0 & 1.21 \\
\hline Mubarak & 0.6 & 0.9 & 2.3 & 1.5 & 2.4 & 0 & 0 & 1.1 & 0.9 & 1.1 & 1.8 & 2 & 1.7 & 0 & 0 & 1.07 \\
\hline NIBGE-8 & 0.7 & 1 & 1.7 & 1.1 & 2.7 & 0 & 0 & 1.03 & 1 & 1.3 & 1.9 & 2.1 & 2.3 & 0 & 0 & 1.23 \\
\hline NIBGE-9 & 0.7 & 0.9 & 1.7 & 1.2 & 3.3 & 0 & 0 & 1.11 & 1 & 1.3 & 1.8 & 2.1 & 2.1 & 0 & 0 & 1.19 \\
\hline RH-668 & 0.8 & 0.9 & 1.8 & 1.4 & 3.5 & 0 & 0 & 1.2 & 1 & 1.3 & 1.8 & 2.1 & 2.3 & 0 & 0 & 1.21 \\
\hline Mean & 0.73 & 0.88 & 1.73 & 1.39 & 2.93 & 0 & 0 & 1.09 & 0.94 & 1.21 & 1.76 & 2.06 & 1.83 & 0 & 0 & 1.12 \\
\hline Alpha 0.05 & & Stanc & ard Err & or for & Compa & irison $=$ & $=0.022$ & & & & & $\mathrm{LSD}=$ & 0.0785 & & & \\
\hline
\end{tabular}

\section{Root length index}

Analysis of data on root length revealed that root length index at both growth stages was highest at osmotic potential of $-0.148 \mathrm{MPa}$ (10\% PEG-6000 concentration) followed by $-0.05 \mathrm{MPa}$ (5\% PEG-6000 concentration) while the least was recorded at -0.846 MPa (27\% PEG-6000 concentration). Maximum mean root length index was recorded for genotype NIBGE8 followed by NIBGE-9 while minimum root length index was recorded for genotype Mubarak at both developmental stages as well as at all concentrations of PEG-6000. At all levels of PEG-6000 (0.0, -0.05, $0.148,-0.295,-0.491,-0.735$ and $-0.846 \mathrm{MPa}$ ) mean root length index recorded on $12^{\text {th }}$ DAS were 712.5 , $726.81,980.81,521.62,130.5,1.18$ and 0 while
$1123.12,1132.12,1197.5,797.62,219.18,3.62$ and 0 , respectively were recorded on $18^{\text {th }}$ DAS (Table-7).

\section{Shoot vigor index}

The results of data analysis on mean shoot vigor index indicated that highest shoot vigor index was observed at both growth stages in NIBGE-8 followed by NIBGE-9 and least was recorded in Mubarak. At PEG6000 concentrations $0,5,10,15,20,25$ and $27 \%$ mean values recorded for shoot vigor index on $12^{\text {th }}$ DAS were $971.87,812.12,570.25,388.5,43.56,0$, and 0 , respectively while at $18^{\text {th }}$ DAS recorded mean values for shoot vigor index were 1198.12, 924.12, 677.37, $378.5,105.75,0$, and 0 , respectively (Table-8). 
Muhammad Riaz Gondal et al.

Table-7. Effect of different concentrations of PEG-6000 on cotton root length index at different growth stages

\begin{tabular}{|c|c|c|c|c|c|c|c|c|c|c|c|c|c|c|c|c|}
\hline \multirow{2}{*}{ Genotype } & \multicolumn{8}{|c|}{ PEG-6000 concentration on $12^{\text {th }}$ DAS } & \multicolumn{8}{|c|}{ PEG-6000 concentration on $18^{\text {th }}$ DAS } \\
\hline & $0 \%$ & $5 \%$ & $10 \%$ & $15 \%$ & $20 \%$ & $25 \%$ & $27 \%$ & Mean & 0\% & $5 \%$ & $10 \%$ & $15 \%$ & $20 \%$ & $25 \%$ & $27 \%$ & Mean \\
\hline BH-201 & 840 & 837 & 1096 & 630 & 200 & 3 & 0 & 515 & 1220 & 1224 & 1256 & 910 & 228 & 6 & 0 & 692 \\
\hline CEMB-55 & 630 & 576 & 888 & 483 & 100 & 0 & 0 & 382 & 1080 & 968 & 1144 & 742 & 160 & 0 & 0 & 585 \\
\hline CEMB-88 & 530 & 585 & 808 & 427 & 76 & 1 & 0 & 347 & 940 & 981 & 1040 & 637 & 180 & 2 & 0 & 540 \\
\hline CIM-602 & 820 & 728 & 910 & 516 & 135 & 0 & 0 & 444 & 1210 & 1080 & 1085 & 786 & 201 & 0 & 0 & 623 \\
\hline CIM-625 & 800 & 712 & 1032 & 498 & 88 & 0 & 0 & 447 & 1200 & 1064 & 1240 & 774 & 142 & 0 & 0 & 631 \\
\hline CIM-632 & 750 & 738 & 976 & 567 & 160 & 1 & 0 & 456 & 1140 & 1152 & 1200 & 882 & 280 & 2 & 0 & 665 \\
\hline CYTO-179 & 570 & 621 & 864 & 396 & 44 & 0 & 0 & 356 & 1020 & 1053 & 1080 & 588 & 94 & 0 & 0 & 548 \\
\hline CYTO-313 & 590 & 560 & 777 & 335 & 48 & 0 & 0 & 330 & 1030 & 936 & 966 & 505 & 108 & 0 & 0 & 506 \\
\hline DEEBEL & 550 & 680 & 945 & 504 & 100 & 2 & 0 & 397 & 990 & 1140 & 1206 & 752 & 225 & 6 & 0 & 617 \\
\hline FH-142 & 770 & 756 & 984 & 574 & 123 & 0 & 0 & 458 & 1140 & 1161 & 1208 & 868 & 186 & 0 & 0 & 652 \\
\hline FH-152 & 750 & 729 & 960 & 567 & 156 & 0 & 0 & 452 & 1130 & 1116 & 1176 & 840 & 236 & 0 & 0 & 643 \\
\hline FH-326 & 790 & 783 & 1016 & 581 & 168 & 0 & 0 & 477 & 1170 & 1179 & 1224 & 896 & 260 & 0 & 0 & 676 \\
\hline Mubarak & 470 & 464 & 693 & 270 & 24 & 0 & 0 & 274 & 930 & 840 & 889 & 435 & 52 & 0 & 0 & 449 \\
\hline NIBGE-8 & 870 & 990 & 1287 & 752 & 270 & 8 & 0 & 597 & 1290 & 1440 & 1539 & 1128 & 475 & 26 & 0 & 843 \\
\hline NIBGE-9 & 850 & 950 & 1251 & 637 & 200 & 2 & 0 & 556 & 1250 & 1400 & 1485 & 1088 & 344 & 9 & 0 & 797 \\
\hline RH-668 & 820 & 920 & 1206 & 609 & 196 & 2 & 0 & 536 & 1230 & 1380 & 1422 & 931 & 336 & 7 & 0 & 758 \\
\hline Mean & 713 & 727 & 981 & 522 & 131 & 1.19 & 0 & 439 & 1123 & 1132 & 1198 & 798 & 219 & 3.63 & 0 & 639 \\
\hline Alpha 0.05 & \multicolumn{8}{|c|}{ or for Comparison 3.8114} & \multicolumn{8}{|c|}{13.05} \\
\hline
\end{tabular}

Table-8. Effect of different concentrations of PEG-6000 on cotton shoot vigor index at different growth stages

\begin{tabular}{|c|c|c|c|c|c|c|c|c|c|c|c|c|c|c|c|c|}
\hline \multirow{2}{*}{ Genotype } & \multicolumn{8}{|c|}{ PEG-6000 concentration on $12^{\text {th }}$ DAS } & \multicolumn{8}{|c|}{ PEG-6000 concentration on $18^{\text {th }}$ DAS } \\
\hline & 0\% & $5 \%$ & $10 \%$ & $15 \%$ & $20 \%$ & $25 \%$ & $27 \%$ & Mean & $0 \%$ & $5 \%$ & $10 \%$ & $15 \%$ & $20 \%$ & $25 \%$ & $27 \%$ & Mean \\
\hline BH-201 & 1090 & 891 & 640 & 504 & 64 & 0 & 0 & 456 & 1270 & 981 & 720 & 455 & 156 & 0 & 0 & 512 \\
\hline CEMB-55 & 900 & 696 & 552 & 315 & 36 & 0 & 0 & 357 & 1160 & 800 & 648 & 354 & 92 & 0 & 0 & 436 \\
\hline CEMB-88 & 830 & 621 & 408 & 273 & 28 & 0 & 0 & 309 & 1090 & 855 & 592 & 315 & 76 & 0 & 0 & 418 \\
\hline CIM-602 & 1050 & 792 & 574 & 462 & 54 & 0 & 0 & 419 & 1250 & 872 & 623 & 372 & 105 & 0 & 0 & 460 \\
\hline CIM-625 & 1000 & 752 & 632 & 396 & 30 & 0 & 0 & 401 & 1230 & 856 & 712 & 366 & 68 & 0 & 0 & 462 \\
\hline CIM-632 & 960 & 819 & 600 & 427 & 52 & 0 & 0 & 408 & 1200 & 945 & 688 & 399 & 120 & 0 & 0 & 479 \\
\hline CYTO-179 & 870 & 738 & 512 & 246 & 18 & 0 & 0 & 341 & 1140 & 882 & 608 & 288 & 40 & 0 & 0 & 423 \\
\hline CYTO-313 & 890 & 672 & 462 & 210 & 20 & 0 & 0 & 322 & 1160 & 808 & 588 & 275 & 54 & 0 & 0 & 412 \\
\hline DEEBEL & 850 & 810 & 576 & 328 & 40 & 0 & 0 & 372 & 1120 & 970 & 376 & 100 & & 0 & 0 & 462 \\
\hline FH-142 & 980 & 882 & 576 & 413 & 33 & 0 & 0 & 412 & 1220 & 945 & 704 & 420 & 102 & 0 & 0 & 484 \\
\hline FH-152 & 970 & 873 & 568 & 399 & 40 & 0 & 0 & 407 & 1200 & 918 & 680 & 399 & 124 & 0 & 0 & 474 \\
\hline FH-326 & 990 & 882 & 592 & 427 & 56 & 0 & 0 & 421 & 1210 & 918 & 664 & 378 & 112 & 0 & 0 & 469 \\
\hline Mubarak & 790 & 536 & 308 & 175 & 10 & 0 & 0 & 260 & 1050 & 736 & 497 & 220 & 30 & 0 & 0 & 362 \\
\hline NIBGE-8 & 1190 & 1040 & 747 & 696 & 100 & 0 & 0 & 539 & 1330 & 1130 & 828 & 536 & 205 & 0 & 0 & 576 \\
\hline NIBGE-9 & 1150 & 1010 & 720 & 511 & 60 & 0 & 0 & 493 & 1300 & 1110 & 819 & 455 & 160 & 0 & 0 & 549 \\
\hline RH-668 & 1040 & 980 & 657 & 434 & 56 & 0 & 0 & 452 & 1240 & 1060 & 801 & 448 & 148 & 0 & 0 & 528 \\
\hline Mean & 972 & 812 & 570 & 389 & 43.6 & 0 & 0 & 398 & 1198 & 924 & 677 & 379 & 106 & 0 & 0 & 469 \\
\hline Alpha 0.05 & \multicolumn{8}{|c|}{ Standard Error for Comparison 3.8569} & \multicolumn{8}{|c|}{ LSD 13.209} \\
\hline
\end{tabular}

\section{Discussion}

The current research focused to investigate the drought tolerance in various genotypes of cotton. The data collection and analysis on various plant growth parameters comprised on germination percentage, root and shoot lengths, root to shoot ratio and vigor indices. These parameters were statistically analyzed, and explored thoroughly in comparison with each other and among their interactions with a wide range of 
osmotic potentials. It was found that the PEG-6000 concentration showed inverse relationship with germination percentage i.e., as concentration increases germination decreases. Drop in germination percentage was due to water stress, which alters the cell function and growth. Xue-yan et al., (2008) found that cellular extension and carbohydrates wall production highly altered and inhibited due to water stress. Water stress ultimately decreases cell enlargement due to turgescence reduction (Shalhevet et al., 1995). The concentrations of $25-27 \%$ creates fatal osmotic potential for germination upon which germination stops (Sidari et al., 2008; Khodarahmpour, 2011; Babu et al., 2014; Megha and Mummigatti, 2017). Tsaliki et al. (2019) and Jatoi et al. (2014) also revealed that sprouting of cotton genotypes decreased under increased drought intensity with application of PEG-6000. Lesser PEG applied greater will be germination as adverse effect upon germination is dependent upon the proportion of drought intensity and duration in addition to PEG6000 used.

It was observed that enlargement in the root length continued until PEG-6000 concentration reached at the level of $10 \%$. Sakthivelu et al. (2008); Khodarahmpour (2011) and Jatoi et al. (2014) also reported decrease in root length under various water deficit conditions. This can be due to the reason that under osmotic stress plant separately execute additional photosynthesis for the enlargement and development of root instead of shoots. It facilitates plant to acquire moisture through deep penetration into the soil whereas reduced shoot size decreases transpiration rate (Tonin et al., 2000; Maruti and Katageri, 2015; Megha and Mummigatti, 2017). Long roots play important role in provision of water to plant by extracting water from the deep zone of soil as long roots are found to withdraw more water per unit length of root from moist ground and withdrawal of water reduces with the reduction of soil water potential (Landjeva et al., 2008 and Babu et al., 2014).

Shortening of shoot length could be due to the fact that under drought stress, plant tends to get moisture from the deep zone of soil, for which root size, number of roots, mass of root and adjacent roots became large and expanded which causes ultimate reduction in shoot length. These results are in complete agreement with the findings of Landjeva et al.,(2008); Sakthivelu et al. (2008); Khodarahmpour (2011); Babu et al. (2014) and Megha and Mummigatti (2017) who observed that shoot biomass decreases due to increase in root lenght volume, weight and lateral roots in search of moisture from deep soil layers. Declined length of shoot decreases transpiration rate due to decrease in surface area for water loss (Babu et al., 2014). Xue-yan et al. (2008) discovered that evaluation and selection of cotton genotypes can be carried out easily and rapidly for drought tolerance by the modification of osmotic conditions by means of PEG-6000. He subjected some cotton genotypes to artificial drought stress conditions for 12 hours by utilizing different concentrations of PEG at sprouting, seedling-, cotyledon- and leaf formation stages. He observed varied amounts of osmotic stress tolerance and found that shoot development as well as 3-6 leaves formation phases were very crucial with respect to osmotic stress tolerance.

Higher ratios might be due to ultimate increment in mass of roots, which enabled the plant roots to extract more water due to increased photosynthetic activity of plant for the development of higher root biomass. Lower shoot length and biomass assisted in the prevention of higher water losses by decreasing transpiration rate per unit area of shoot. They may change to maintain existence under osmotic stress conditions instead of having contribution in yield (Khodarahmpour, 2011; Babu et al., 2014 and Megha and Mummigatti, 2017). Meneses et al. (2011) reported that osmotic potential below $-0.4 \mathrm{MPa}$ have drastic effects upon seed viability and seedling vigor. Likewise earlier studies; on cotton by Michel and Kaufmann (1973), on cowpea by Ogbonnaya et al. (2003) and on wheat by Landjeva et al. (2008) revealed that genotypes, which were tolerant to drought stress circumstances attains higher root to shoot ratio as compared to the susceptible cultivars. Megha and Mummigatti, (2017) narrated that root length index decreases as osmotic potential increases by using PEG-6000 in susceptible genotypes but shoot vigor index showed inverse relationship with PEG6000 concentration. The reduction in shoot vigor index is probably due to lengthy root and shoot; smaller number of leaves and reduced seedling length. Xue-yan et al. (2008) also reported the similar results.

\section{Conclusion}

From the current investigation, it is concluded that various cotton varieties showed different behavior against stress. Some varieties had significant tolerance against drought stress generated by PEG-6000 at some levels. Water deficiency highly effects the survival of 
seed and seedling development at different osmotic conditions. On basis of findings of this study, it is concluded that genotype NIBGE-8 was highly osmotic stress tolerant whereas cultivar Mubarak was highly sensitive to water stress. Furthermore, the results of the study revealed significance of PEG-6000 as synthetic stress inducer for quick evaluation and screening of drought tolerant cotton genotypes that can play key role in cotton breeding activities.

\section{Acknowledgment}

The authors are obliged of the management of Gomal University D.I. Khan who provided the facilities of laboratory to conduct the experiment.

Disclaimer: None.

Conflict of Interest: None.

Source of Funding: None.

\section{References}

Abdul- Baki AA and Anderson JD, 1973. Vigor determination in soybean seed by multiple criteria. Crop Sci. 13:630-633.

Allen RG, Pereira LS, Raes D and Smith M, 1998. Crop evapotranspiration-Guidelines for computing crop water requirements-FAO Irrigation and drainage paper 56. FAO, Rome. 300: D05109.

Babu A, Patil B and Pawar K, 2014. Evaluation of cotton genotypes for drought tolerance using PEG-6000 water stress by slanting glass plate technique. The Bioscan. 9:1419-1424.

Boman R and Lemon R, 2006. Drought management strategies related to production, management, and marketing of cotton in Texas. Texas A\&M Agri. Life Extension Service. Texas A\&M Univ., College Station, TX. Available online at http://varietytesting.tamu.edu/forages/drought/Co tton_drought_final_RL_RB_06.pdf (verified 13 July 2014).1-4.

Dabbert T and Gore MA, 2014. Challenges and perspectives on improving heat and drought stress resilience in cotton. J. Cotton Sci. 18:393-409.

Gomez KA and Gomez AA, 1984. Statistical procedures for agricultural research, John Wiley \& Sons, USA.

Economic Survey of Pakistan 2019-20, 2020. Economic Advisor's Wing, Finance Division, Government of Pakistan, Islamabad, Pakistan.
Jatoi SA, Latif MM, Arif M, Ahson M and Siddiqui S, 2014. Comparative assessment of wheat landraces against polyethylene glycol simulated drought stress. Sci. Technol and Dev. 33:1-6.

Khodarahmpour Z, 2011. Effect of drought stress induced by polyethylene glycol (PEG) on germination indices in corn (Zea mays L.) hybrids. Afr. J. Biotechnol. 10:18222-18227.

Landjeva S, Neumann K, Lohwasser U and Börner A, 2008. Molecular mapping of genomic regions associated with wheat seedling growth under osmotic stress. Biologia Plantarum. 52:259-266.

Maruti L and Katageri I, 2015. Genetic influence of root traits of cotton (Gossypium hirsutum L.) on moisture stress tolerance. Karnataka J. Agric. Sci. 28:454-458.

McWilliams D, 2003. Drought strategies for cotton. p. 1-5 In New Mexico State University Cooperative Extension Service. Circular 582. College of Agriculture and Home Economics, New Mexico State Univ., Las Cruces, NM. Available online at http://aces.nmsu.edu/pubs/_circulars/ CR582.pdf (verified 13 July 2014).

Megha B and Mummigatti U, 2017. Screening of hirsutum cotton genotypes for drought tolerance under different osmotic potential and field capacities. Int. J. Bio-res. Stress Manage. 8:299308.

Meneses CHSG, Bruno RdLA, Fernandes PD, Pereira WE, Lima LHGdM, Lima MMdA and Vidal MS, 2011. Germination of cotton cultivar seed under water stress induced by polyethyleneglycol-6000. Scientia Agricola. 68:131-138.

Michel BE and Kaufmann MR, 1973. The osmotic potential of polyethylene glycol 6000. Plant Physiol. 51:914-916.

Ogbonnaya C, Sarr B, Brou C, Diouf O, Diop N and Roy- Macauley H, 2003. Selection of cowpea genotypes in hydroponics, pots, and field for drought tolerance. Crop Sci. 43:1114-1120.

Pace P, Cralle HT, El-Halawany SH, Cothren JT and Senseman SA, 1999. Drought-induced changes in shoot and root growth of young cotton plants. J. Cotton Sci. 3:183-187.

Riaz M, Farooq J, Sakhawat G, Mahmood A, Sadiq M and Yaseen M, 2013. Genotypic variability for root/shoot parameters under water stress in some advanced lines of cotton (Gossypium hirsutum L.). Genet. Mol. Res. 12:552-561.

Romagosa I and Fox PN, 1993. Genotype $\times$ environment interaction and adaptation. J. Plant 
Breed. DOI: https://doi.org/10.1007/978-94-0111524-7_23

Sakthivelu G, Devi MA, Giridhar P, Rajasekaran T, Ravishankar G, Nedev T and Kosturkova G, 2008. Drought-induced alterations in growth, osmotic potential and in vitro regeneration of soybean cultivars. Gen. Appl. Plant Physiol. 34:103-112.

Shalhevet J, Huck MG and Schroeder BP, 1995. Root and shoot growth responses to salinity in maize and soybean. Agron. J. 87:512-516.

Sidari M, Santonoceto C, Anastasi U, Preiti G and Muscolo A, 2008. Variations in four genotypes of lentil under $\mathrm{NaCl}$-salinity stress. Am. J. Agric. Biol. Sci. 3:410-416.

Steel RG, 1997. Pinciples and procedures of statistics a biometrical approach. 3rd ed. McGraw-Hill, Boston, USA. 666p.

Tonin G, Carvalho N, Kronka S and Ferraudo A, 2000. Culture systems, velvet bean and mineral fertilization influence on maize seed physiological quality. Revista Brasileira de Sementes. 22:276279.

Tsaliki E, Xanthopoulos F, Kechagia U and Leloudis C, 2019. Evaluation of germination ability of cotton cultivars (Gossypium hirsutum L.) under artificial stress conditions. Agric. Sci. Pract. 4: 48.
Wen Y, Rowland DL, Piccinni G, Cothren JT, Leskovar DI, Kemanian AR and Woodard JD, 2013. Lint yield, lint quality, and economic returns of cotton production under traditional and regulated deficit irrigation schemes in Southwest Texas. J. Cotton Sci. 17:10-22.

Xue-yan Z, Chuan-liang L, Jun-juan W, Fu-Guang L and Wu-wei Y, 2008. Drought-tolerance evaluation of cotton with PEG water-stress method. Cotton Sci. 20:56-61.

\section{Contribution of Authors}

Gondal MR: Planned and managed the study, supervised data collection and analysis

Saleem MY: Conceived idea, designed research methodology, data collected and first draft write up Rizvi SA: Interpreted data and final editing \& approval of manuscript

Riaz A: Literature review and manuscript write up Naseem W \& Muhammad G: Literature review, data analysis and interpretation

Hayat S: Research execution and data collection Iqbal M: Data analysis, final editing \& approval of manuscript 